\title{
Mouse model of Devil FacialTumour Disease establishes that an effective immune response can be generated against the cancer cells
}

\author{
Terry L. Pinfold ${ }^{1}$ *, Gabriella K. Brown ${ }^{1}$, Silvana S. Bettiol ${ }^{2}$ and Gregory M. Woods ${ }^{1}$ \\ Menzies Research Institute Tasmania, University of Tasmania, Hobart, TAS, Australia \\ 2 School of Medicine, University of Tasmania, Hobart, TAS, Australia
}

Edited by:

Bibhash Mukhopadhyay, Johnson \& Johnson, USA

\section{Reviewed by:}

Chris Schmidt, Queensland Institute of Medical Research, Australia Paul Joseph Neeson, Peter

MacCallum Cancer Centre, Australia

*Correspondence:

Terry L. Pinfold, Menzies Research Institute Tasmania, University of

Tasmania, 17 Liverpool Street, Hobart, TAS 7000, Australia

e-mail: tpinfold@utas.edu.au

\begin{abstract}
The largest carnivorous marsupial in Australia, the Tasmanian devil (Sarcophilus harrisii) is facing extinction in the wild due to a transmissible cancer known as Devil Facial Tumour Disease (DFTD). DFTD is a clonal cell line transmitted from host to host with $100 \%$ mortality and no known immunity. While it was first considered that low genetic diversity of the population of devils enabled the allograft transmission of DFTD recent evidence reveals that genetically diverse animals succumb to the disease. The lack of an immune response against the DFTD tumor cells may be due to a lack of immunogenicity of the tumor cells. This could facilitate transmission between devils. To test immunogenicity, mice were injected with viable DFTD cells and anti-DFTD immune responses analyzed. A range of antibody isotypes against DFTD cells was detected, indicating that as DFTD cells can induce an immune response they are immunogenic. This was supported by cytokine production, when splenocytes from mice injected with DFTD cells were cultured in vitro with DFTD cells and the supernatant analyzed. There was a significant production of IFN- $\gamma$ and TNF- $\alpha$ following the first injection with DFTD cells and a significant production of IL-6 and IL-10 following the second injection. Splenocytes from naïve or immunized mice killed DFTD cells in in vitro cytotoxicity assays. Thus, they are also targets for immunological destruction. We conclude that as an immune response can be generated against DFTD cells they would be suitable targets for a vaccine.
\end{abstract}

Keywords: Tasmanian devil, transmissible cancer, mouse model, immunogenicity, Devil Facial Tumour Disease

\section{INTRODUCTION}

The Tasmanian devil (Sarcophilus harrisii) faces possible extinction in the wild due to a transmissible cancer known as Devil Facial Tumour Disease (DFTD). Genetic and chromosomal research has provided convincing evidence that the malignant neoplasm originated in an individual female Tasmanian devil (1). While it was first considered that low genetic diversity of the population of devils, from which the DFTD founder was derived, enabled the establishment of DFTD throughout subsequent devil populations $(2,3)$, recent evidence reveals that genetically diverse animals are prone to the disease (4).

The unique and conserved chromosomal rearrangements of DFTD cells compared to the host's negate the possibility of transmission from a viral or bacterial agent, pollutants or toxins in the environment. DFTD is described, alongside the canine transmissible venereal tumor (CTVT), as a clonal cell line immortalized as a parasitic infectious allograft (5). Not only did the DFTD cancer cells evade the original host's immune system but the immune systems of subsequent devils. The cancer cells are transmitted through facial biting of successive hosts $(1,6,7)$.

A lack of immunogenicity is one possible mechanism for DFTD cells to evade the immune system. A failure to present tumor antigens to the immune system would facilitate transmission between devils. Siddle et al. (8) revealed that DFTD cells do not express cell surface MHC molecules in vitro or in vivo. The genes essential to the antigen-processing pathway, such as $\beta_{2}$-microglobulin and transporters associated with antigen processing are downregulated. The loss of gene expression is not due to structural mutations, but to regulatory changes including epigenetic deacetylation of histones (8). By down-regulating MHC, the tumor cells remain invisible to the devils' immune system. But even in the absence of MHC expression, there should be enough protein differences to induce an immune response following allogenic transfer.

In order to develop an effective vaccine or immunotherapy, it is important to understand the mechanisms that shield this tumor from immunosurveillance. Ideally, an immunological study of an allograft tumor in the host species is necessary, but in the case of the Tasmanian devil conducting large scale immunological experiments are not possible due to the endangered species status. Therefore, since it is widely accepted that mouse models provide valuable insights into the study of human cancers our investigation exploits a mouse model to study DFTD. The particular advantage of a mouse model is the readily available antibodies to detect mouse immune system responses while there is a paucity of equivalent antibodies currently available for the Tasmanian devil immune system. 
To determine if DFTD cells are immunogenic and therefore potential targets for immunotherapy, we used a mouse model developed in our laboratory to examine DFTD. We have previously shown that the tumors successfully implant in immunocompromised NOD/SCID mice but do not implant in immunocompetent $\mathrm{BALB} / \mathrm{c}$ and $\mathrm{C} 57 \mathrm{BL} / 6$ mice. The aim was to determine if this rejection by immunocompetent mice was an active immunological response and not due to other factors such as preformed antibodies commonly associated with xenogeneic graft rejection. This was performed by examining specific antibody, cytokine, and cell mediated cytotoxicity responses to the DFTD xenograft.

In addition, this xenograft model was used to investigate the possibility that if DFTD cells are immunogenic they may polarize the murine immune system toward a $\mathrm{T}_{\mathrm{H}} 2$ response. This is a mechanism exploited by tumor cells to subvert anti-tumor immune responses toward ineffective humoral responses (9).

\section{MATERIALS AND METHODS \\ MICE}

All mice were obtained from the University of Tasmania Central Animal House and housed in a pathogen free environment and provided with food and water ad libitum. The two strains used were $\mathrm{C} 57 \mathrm{BL} / 6$ and $\mathrm{BALB} / \mathrm{c}$. The mice were at least 5 weeks old at commencement of experimental treatments.

All animal experiments were performed with approval of the Animal Ethics Committee of University of Tasmania (UTAS), approval numbers A0010231 and A0010888.

\section{CELL LINES AND CULTURE MEDIA}

Devil Facial Tumour Disease cell line, C5065, was obtained from stocks stored in liquid nitrogen at the UTAS. The cell line was established from primary tumor biopsy samples taken under the approval of the Animal Ethics Committee of Tasmania's Park and Wildlife Services (permit numbers 33/2004-5 and 32/2005-6) and provided by A.-M. Pearse and K. Swift, Tasmanian Department of Primary Industries, Parks, Wildlife and Environment (DPIPWE). Cells were grown in RPMI-10FCS, which consisted of RPMI-1640 medium (GIBCO, New York, USA) supplemented with $10 \%$ fetal calf serum (FCS) (Bovogen Biological, VIC, Australia), $5 \mathrm{mM} \mathrm{L-}$ glutamine (Sigma-Aldrich, St Louis, MO, USA), and $40 \mathrm{mg} / \mathrm{ml}$ of gentamicin (Pfizer, Bentley Australia). The C5065 cell line culture was maintained in a fully humidified $5 \% \mathrm{CO}_{2}$ incubator at $35^{\circ} \mathrm{C}$. Assays required co-culturing of mouse splenocytes with $\mathrm{C} 5065$ were maintained at $37^{\circ} \mathrm{C}$.

\section{INDIRECT IMMUNOFLUORESCENCE ASSAY FOR DETECTION OF ANTIBODIES BY FLOW CYTOMETRY}

Blood was collected from mice post mortem via cardiac puncture or via mandible bleed with living mice, allowed to clot and the serum was separated by centrifuging. Serum was stored at -20 or $-80^{\circ} \mathrm{C}$ until required. One microliter of each serum sample was incubated with $10^{5}$ DFTD cells in $50 \mu$ l of staining buffer [phosphate-buffered saline (PBS) with 1\% (w/v) bovine serum albumin (BSA) (Roche Diagnostics, Germany) and $0.01 \% \mathrm{NaN}_{3}$ ], on ice for $30 \mathrm{~min}$. Following two washes with PBS, the cells were incubated with $2 \mu \mathrm{g}$ of the relevant anti-mouse immunoglobulin Alexa-fluor conjugated antibody (Life Technologies, USA, catalog numbers IgG A-31553, IgG 1 A-21121, IgG $2 \mathrm{a}$ A-21136, IgG $2 \mathrm{~b}$ A21146, IgG 3 A-21151, IgM A-21042) in $100 \mu$ l of staining buffer on ice for $30 \mathrm{~min}$. Negative controls included DFTD cells with either no serum or pooled naïve serum. Following three washes in PBS, the samples were resuspended in $100 \mu \mathrm{l}$ of PBS for prompt reading on the flow cytometer (BD Canto II, Becton Dickinson, NJ, USA).

The forward scatter, side scatter, thresholds, and PMT voltages were optimized to place the negative mean fluorescence intensity (MFI) for the negative controls at approximately the second decade of the log scale. FCS 4 Express Flow Cytometry software (De nova Software, USA) was used to perform histogram overlays to compare indirect immunofluorescence levels of samples with naïve serum control. The geometric mean of the MFI for immune serum was divided by the geometric mean of the MFI for naïve serum control to give the relative antibody levels.

\section{IMMUNIZATIONS}

Devil Facial Tumour Disease cells in log growth phase were harvested from culture and viability was ascertained by trypan blue exclusion. Cell viability ranged from 50 to $80 \%$. Cells were washed, resuspended in $\mathrm{PBS}$, and $2 \times 10^{6}$ viable cells were injected via the subcutaneous (SC) route or intraperitoneal (IP) route into $\mathrm{C} 57 \mathrm{BL} / 6$ and $\mathrm{BALB} / \mathrm{c}$ mice. With multiple sites SC injections, the cells were divided between multiple sites as stated. No adjuvants were used.

\section{MOUSE MONONUCLEAR CELLS FOR IN VITRO CYTOKINE AND CYTOTOXICITY ASSAYS}

Spleens were removed from naïve or IP immunized mice. Lymph nodes were obtained from SC immunized mice. Mononuclear cells (MNC) were obtained by pressing spleens or lymph nodes through $40 \mu \mathrm{m}$ cell strainers (BD Falcon, USA) and then separating them by density-gradient centrifugation on Histopaque-1083 gradient (Sigma-Aldrich, USA) following the manufacturer's protocol. The MNC were washed twice with PBS and resuspended in RPMI-10FCS for use in in vitro cytotoxicity and cytokine assays.

\section{IN VITRO CYTOKINE CULTURES FROM SPLENOCYTE SUPERNATANTS}

Mononuclear cells were prepared as described above and resuspended in RPMI-10FCS at $10^{7}$ cells/ml. DFTD cells were harvested and resuspended at $10^{5}$ cells/ml in RPMI-10FCS. One hundred microliters each of DFTD and lymphocyte suspensions were combined in V-bottomed 96 well plates (Greiner Bio-one, Frickenhausen, Germany). Control wells were also prepared in the same plate by combining $100 \mu \mathrm{l}$ RPMI with $100 \mu \mathrm{l}$ of either DFTD or $\mathrm{MNC}$ as indicated. The plates were covered by lids and incubated for $72 \mathrm{~h}$ at $37^{\circ} \mathrm{C}$ with $5 \% \mathrm{CO}_{2}$. The plates were centrifuged to pellet the cells and the supernatant collected and stored at $-20^{\circ} \mathrm{C}$ until required. Supernatant samples were assayed individually for cytokine activity using BD Biosciences CBA $\mathrm{T}_{\mathrm{H}} 1, \mathrm{~T}_{\mathrm{H}} 2, \mathrm{~T}_{\mathrm{H}} 17$ micro-bead array kit and FACS array software as per manufacturer's instruction (BD Biosciences, Cat \# 560485). In brief, $50 \mu \mathrm{l}$ of each supernatant sample and cytokine standards were incubated with mixed capture beads and PE detection reagent for $3 \mathrm{~h}$, washed, resuspended, and run on the BD Canto II, flow cytometer. Cytokine data were analyzed using FCAP Array software (BD Bioscience, San Jose, CA, USA). 


\section{CYTOTOXICITY ASSAY USING IMMUNOFLUORESCENCE DOUBLE STAINING PROTOCOL}

DFTD ( $10^{7}$; target cells) were labeled with 5(6)-Carboxyfluorescein diacetate $N$-succinimidyl ester (CFSE) by incubating with $2 \mu \mathrm{l}$ of $5 \mathrm{mM}$ CFSE for $30 \mathrm{~min}$ at $23^{\circ} \mathrm{C}$ on shaker plate protected from light. Cells were washed twice and resuspended at $10^{5}$ cells $/ \mathrm{ml}$ in RPMI-10FCS.

Mononuclear cells were resuspended in RPMI-10FCS at $10^{7}$ cells/ml. A V-bottomed 96 well plate (Greiner Bio-one, Frickenhausen, Germany) was prepared with $100 \mu \mathrm{l}$ serial dilutions of the MNC to provide effector ratios of 100:1, 50:1, 25:1, 12:1, 6:1, and 3:1 with $10^{4}$ target cells.

The cytotoxicity assays were performed using four replicate samples and incubating for $18 \mathrm{~h}$ at $37^{\circ} \mathrm{C}$ in a fully humidified $5 \%$ $\mathrm{CO}_{2}$ incubator. The plates were centrifuged for $5 \mathrm{~min}$ at $500 \mathrm{~g}$, supernatant removed, pellet resuspended in $100 \mu \mathrm{l}$ PBS $+1 \mu \mathrm{l}$ propidium iodide (PI), and read on a BD Canto II flow cytometer with the High Throughput Sampler unit directly recovering samples from the wells.

Flow cytometry data analysis was performed using Flowing Software (Turku Centre for Biotechnology, Finland), or FCS 4 Express Flow Cytometry (De Nova Software, USA), with further analysis and graphs produced in Microsoft Excel 2007 and GraphPad Prism 5.

Cells positive for CFSE and PI were identified as dead target cells whereas cells positive for CFSE, but negative for PI, were identified as viable target cells. Cytotoxicity per well was calculated as dead target cells divided by the sum of dead and viable target cells and expressed as a percentage. The average cytotoxicity for each effector target ratio was calculated and the standard error of the mean (SEM) values was used for the error bars.

\section{STATISTICAL ANALYSES}

Quantitative data comparing two groups are expressed as the mean \pm SEM and $P$-values calculated using Student's unpaired two tailed $t$-test. Quantitative data comparing more than two groups are expressed as the mean with the probability calculated by one-way ANOVA followed by Dunnett's test.

\section{RESULTS}

\section{NAÏVE BALB/c AND C57/BL6 MICE HAVE BARELY DETECTABLE LEVELS OF PREFORMED ANTIBODIES BUT FOLLOWING IMMUNIZATON HAVE HIGH LEVELS OF SPECIFIC ANTIBODIES}

There was no evidence for substantial levels of preformed antibodies as serum from naïve mice showed minimal binding to DFTD cells. This was the case for both IgG and IgM. As shown in Figure 1, flow cytometry profiles of serum from three representative naïve BALB/c and C57/BL6 mice show barely detectable levels of preformed antibodies. When immunized intraperitoneally, both strains of mice had considerable increases in levels of specific antibodies to DFTD cells. As future experiments were aimed at evaluating specific immunity following DFTD cell immunization, the results were summarized as histograms rather than displaying multiple flow cytometry plots. The geometric mean of the MFI of the serum from immunized mice was divided by the geometric mean of the MFI of the serum from naïve mice.

\section{SUBCUTANEOUS INJECTIONS OF BALB/c MICE WITH DFTD CELLS INDUCES ANTIBODY PRODUCTION}

$\mathrm{BALB} / \mathrm{c}$ mice were twice injected subcutaneously with viable DFTD cells, which were obtained from cell culture. The objective was to determine if living DFTD cells could elicit an antibody response. Flow cytometry was used to analyze serum for IgG and IgM antibodies specific to DFTD surface antigens. All the IgG isotypes tested for were detected, but IgM was not detected (Figure 2), thus indicating that DFTD cells can induce an antibody response. The responses to single site SC injections were compared with multiple site SC injections to determine if multiple site injections with the same number of total cells produced a greater response. Multiple site SC injections produced greater total IgG $(P<0.05$; unpaired Student's $t$-test $)$ and IgG1 $(P<0.01$; unpaired Student's $t$-test $)$ responses than injecting the same number of DFTD cells into a single site (Figure 2).

\section{INTRAPERITONEAL INJECTIONS OF C57BL/6 MICE WITH DFTD CELLS INDUCE GREATER ANTIBODY RESPONSES THAN SC INJECTIONS}

C57BL/6 mice were intraperitoneally or subcutaneously injected twice with viable DFTD cells. The objective was to determine, which route produced a better antibody response. Results in Figure 3 show that the IP route produced both IgM and IgG antibodies. The responses following the IP injections were stronger than the SC route for IgM $(P<0.01$; unpaired Student's $t$-test $)$, total IgG, IgG1, IgG2a $(P<0.001$; unpaired Student's $t$-tests $)$, and IgG3 ( $P<0.05$; unpaired Student's $t$-test).

\section{A SINGLE INTRAPERITONEAL INJECTION OF C57BL/6 MICE WITH DFTD CELLS INDUCES BOTH IgM AND IgG ANTIBODY RESPONSES WITHIN 7 DAYS}

Having established that immunization with DFTD cells induces strong IgG responses following two injections, we next determined if IgG and IgM responses could be detected after a single injection. Results in Figure 4 show 4 days was insufficient to generate optimum levels of antibody in the serum with IgM detected in only two of the four mice and IgG detected in only one of these two mice. By 7 days all mice produced both IgM and IgG antibodies with the predominant isotype being $\operatorname{IgG}(P<0.05$; Student's unpaired $t$-test) (Figure 4).

\section{SECONDARY ANTIBODY RESPONSES DEMONSTRATE ENHANCED MEMORY RESPONSES FOR IgG BUT NOT IgM}

Responses to secondary immunizations following 95 days showed enhanced responses in IgG1, IgG2b, and IgG3 antibodies but not $\operatorname{IgM}$ (Figures 4 and 5). The enhanced response is consistent with a primed immune system memory response because it was evident 95 days after the primary immunization.

\section{INTRAPERITONEAL INJECTIONS OF DFTD CELLS DO NOT INDUCE GREATER CYTOTOXICITY IN SPLENOCYTES THAN THOSE OBTAINED FROM NAÏVE MICE}

As IP injections of DFTD cells produced the best antibody response, we used this route to determine if an enhanced cytotoxic response could be induced against DFTD cells. Splenocytes from naïve C57BL/6 mice demonstrated dose response cytotoxicity 


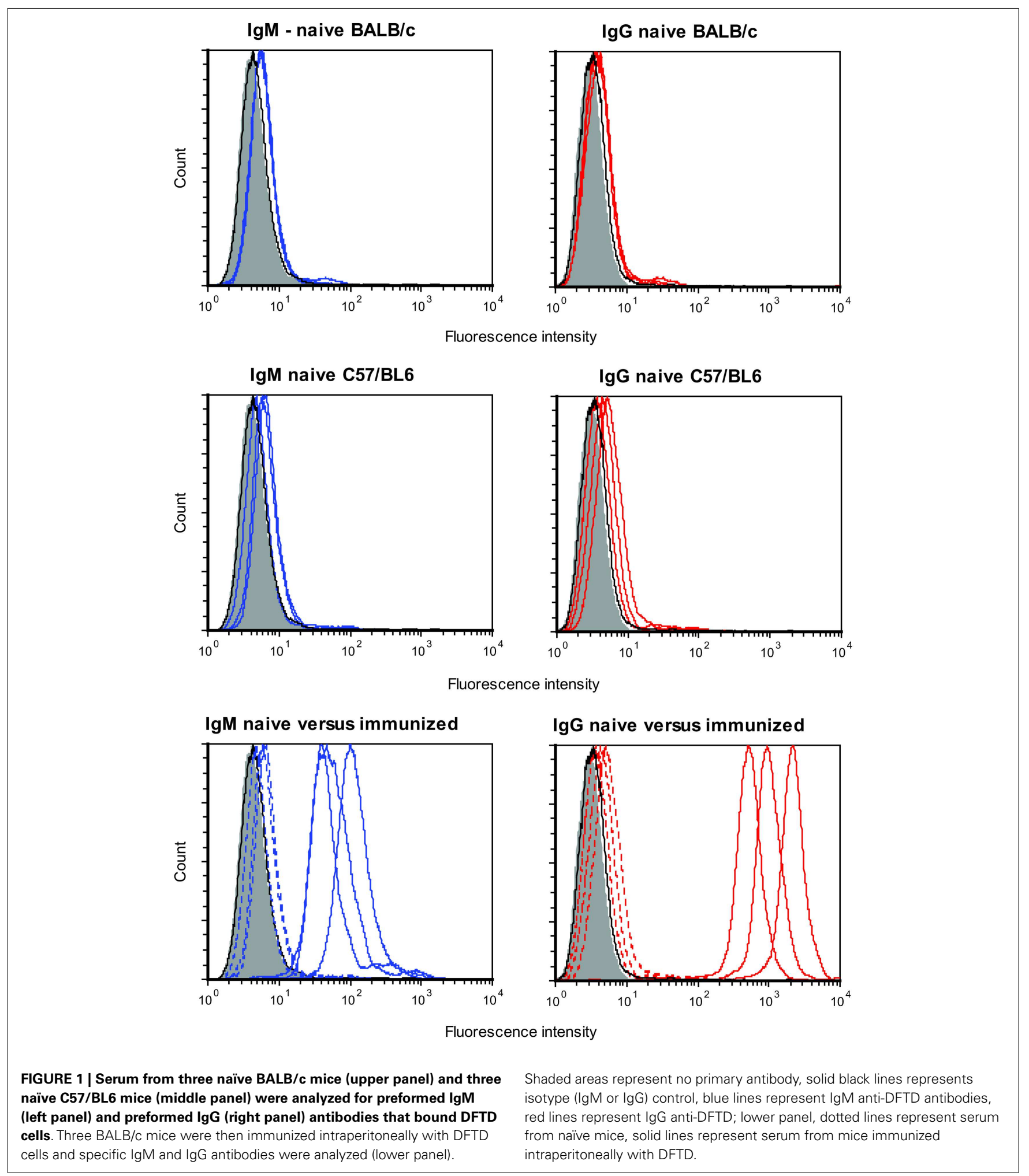

against DFTD cells following $18 \mathrm{~h}$ incubation (Figure 6). This was not improved following two IP injections with DFTD cells, indicating that unprimed cells of the immune system mediated the cytotoxicity.

IMMUNIZATION WITH DFTD CELLS DOES NOT BIAS ANTIBODY OR CYTOKINE RESPONSES TOWARD A TH2 PROFILE

Since tumor cells can weaken anti-tumor immune responses by skewing them toward less effective $\mathrm{T}_{\mathrm{H}} 2$ responses, we examined 

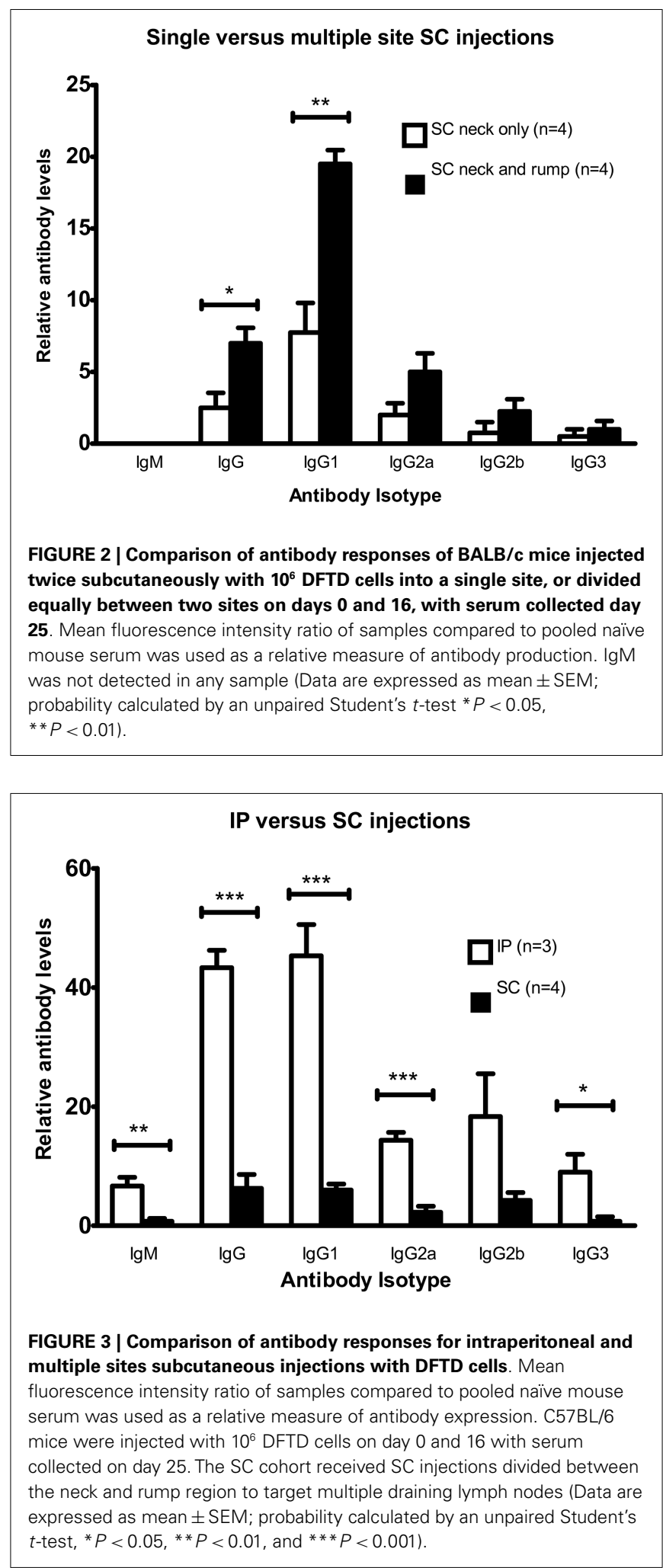

the antibody and cytokine profiles for such a bias. Two strains of mice were compared because of their reportedly opposing $\mathrm{T}_{\mathrm{H}} 1(\mathrm{C} 57 \mathrm{BL} / 6)$ or $\mathrm{T}_{\mathrm{H}} 2(\mathrm{BALB} / \mathrm{c})$ dominated immune responses $(10,11)$.

\section{Antibody response day 4 and 7 after DFTD immunisation}

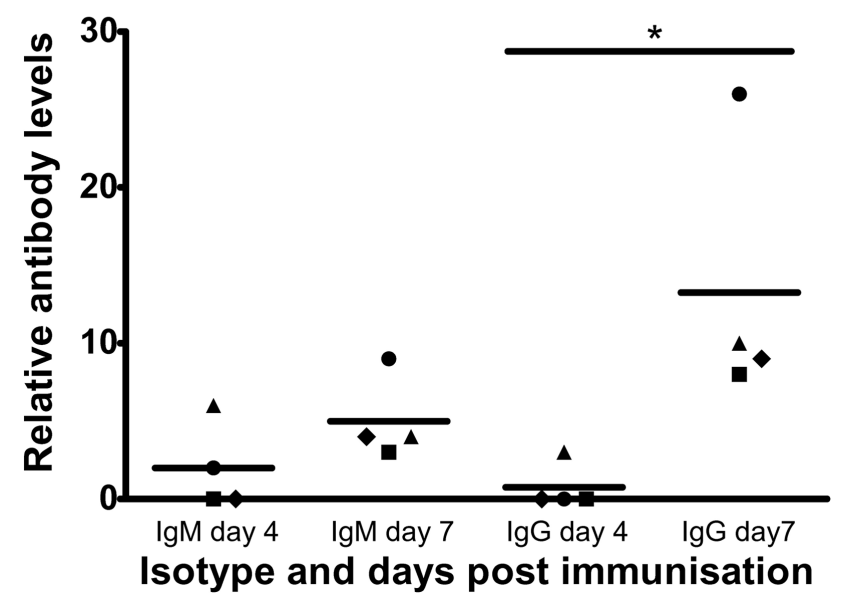

FIGURE 4 | C57BL/6 mice were injected IP with $10^{6}$ DFTD cells and serum was collected $\mathbf{4}$ and $\mathbf{7}$ days post injection. All samples were analyzed in the same flow cytometry experiment to permit direct comparison of mouse anti-DFTD IgG and IgM in the serum. Mean fluorescence intensity ratio of samples compared to pooled naïve mouse serum was used as a relative measure of antibody expression. There was no significant difference in the mean of IgM expression for all four mice (represented by different plot symbols) compared on days 4 and 7 post injection. There was a significant difference of $\mathrm{lgG}$ expression between days 4 and 7 ( $P<0.05$; probability calculated by a paired Student's $t$-test).

Mice were injected intraperitoneally twice with DFTD cells and serum was collected 7-8 days following the second immunization. Levels of IgM, the $\mathrm{T}_{\mathrm{H}} 2$ antibody, IgG1, and the $\mathrm{T}_{\mathrm{H}} 1$ antibody IgG3 were not significantly different between the two strains (Figure 7). Of the other $\mathrm{T}_{\mathrm{H}} 1$ antibodies, $\mathrm{BALB} / \mathrm{c}$ mice expressed higher levels of IgG2a and lower levels of IgG2b compared to C57BL/6 $(P<0.05$; unpaired Student's $t$-test $)$.

As up-regulation of certain cytokines in the tumor microenvironment can enhance or suppress tumor rejection, we next assessed the cytokine production of splenocytes from naïve mice, mice given a single IP injection of DFTD and mice given two IP injections of DFTD cells. Splenocytes were obtained from the mice and incubated in vitro for $72 \mathrm{~h}$ with DFTD cells and the supernatant analyzed for cytokines. Cytokine levels expressed into the growth media were then measured.

Splenocytes from non-immunized mice did not produce detectable cytokines. Evidence for IFN- $\gamma$, TNF, IL-6, and IL-10 production was apparent for splenocytes obtained at 4 and 21 days following a single immunization (Figure 8). These same cytokines were also detected when splenocytes were obtained 5 days after a secondary DFTD immunization.

Of the $\mathrm{T}_{\mathrm{H}} 1$ cytokines, peak levels of IFN- $\gamma$ and TNF- $\alpha$ were detected from splenocytes obtained at day 21 after a single immunization. Peak levels of the $\mathrm{T}_{\mathrm{H}} 2$ cytokine IL-10 were detected following a secondary immunization. The pro-inflammatory cytokine IL-6 followed a similar pattern to IL-10 with peak values occurring after a secondary immunization. IL-4 and IL-12 were 


\section{IgM primary and secondary responses}

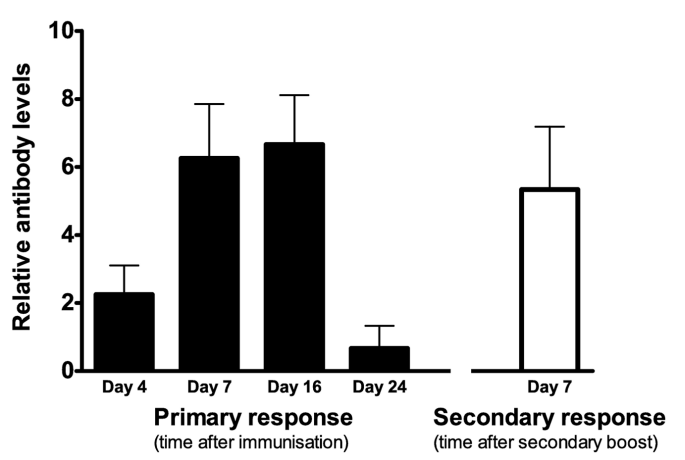

IgG2b primary and secondary responses

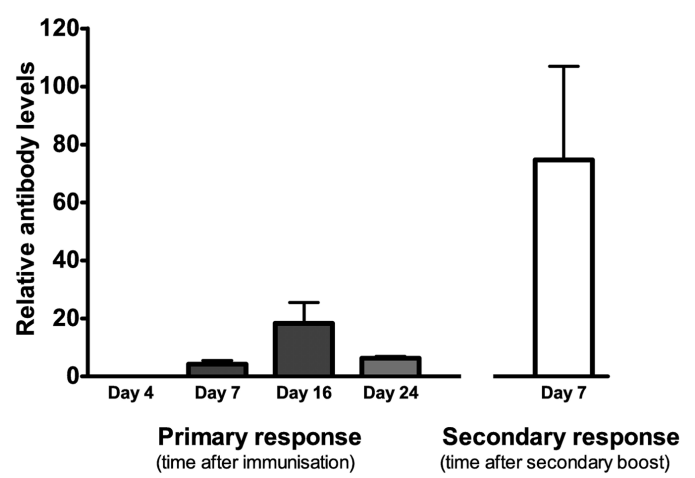

FIGURE 5 | Following a single IP injection serum samples were collected on day 4, 7, 16, and 24 (results shown in black).

Secondary immunization occurred on day 95 and serum was

collected 7 days later (results shown in white). Mean fluorescence

\section{IgG1 primary and secondary responses}

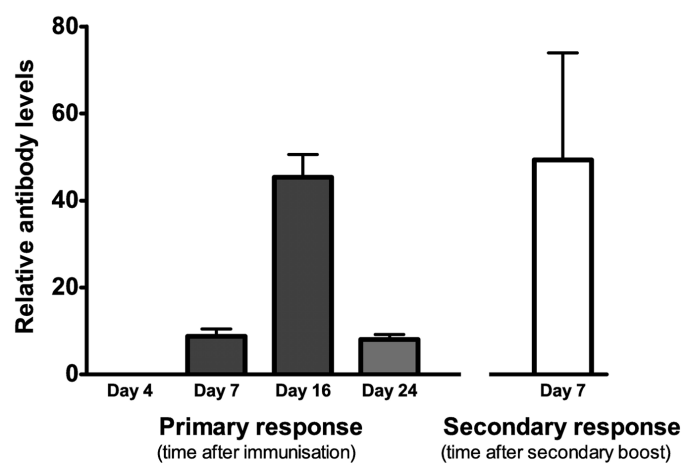

IgG3 primary and secondary responses

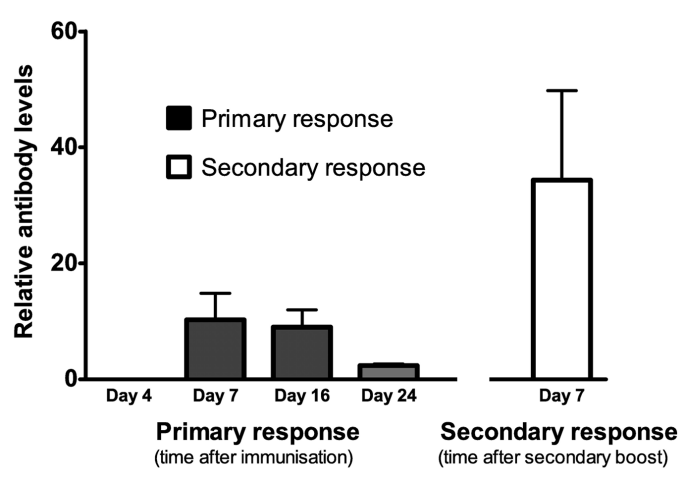

intensity ratio of samples compared to pooled naïve mouse serum was used as a relative measure of antibody expression. Data expressed as mean of three mice \pm SEM except day 4 and 7 primary response $(n=4)$. not produced at detectable levels and are therefore not shown. The antibody and cytokine profiles did not suggest that immunization with DFTD cells polarized the immune response toward a $\mathrm{T}_{\mathrm{H}} 1$ or $\mathrm{T}_{\mathrm{H}} 2$ profile.

\section{IRRADIATED CELLS RETAIN IMMUNOGENICITY WHILE SONICATION OR FREEZE/THAW LYSATES HAVE REDUCED IMMUNOGENICITY}

Inactivated DFTD cells that maintain their immunogenicity are required for vaccine and immunotherapy trials. To determine the best means of achieving this, BALB/c mice were injected twice IP with DFTD cells. The cells were either viable or inactivated by irradiation, sonication, or rapid freeze/thawing. Serum was collected for analysis of anti-DFTD antibodies by flow cytometry. Splenocytes from DFTD immunized mice were co-cultured with DFTD cells and the supernatant analyzed for cytokine expression.

Mice immunized with irradiated cells produced higher levels of DFTD specific antibodies compared to sonicated and freeze/thaw lysates. When cultured in vitro with DFTD cells, splenocytes from mice immunized with sonicated or freeze/thaw lysates produced lower levels of IFN- $\gamma$ and TNF- $\alpha$ compared to splenocytes from mice immunized with irradiated cells (Figure 9). Irradiation of cells is the better method for inactivation because immunogenicity is maintained.

\section{DISCUSSION}

Devil Facial Tumour Disease is a cancer that is transmitted from host to host with $100 \%$ mortality and no known immunity. Lack of surface MHC-I expression on the DFTD cancer cells (8) explains the lack of allo-recognition. An inability to trigger an immune response suggests that DFTD cells are non-immunogenic and could be imperceptible to any immune system. This study focused on the fundamental question of whether DFTD cells are immunogenic and therefore it should be possible to generate an immune response against DFTD tumor cells. As access to Tasmanian devils is limited due to their endangered status and there are limited reagents such as monoclonal antibodies, we conducted this study in our mouse model of DFTD (12).

The ability to establish DFTD xenografts in immunocompromised NOD/SCID (12) mice but not in immunocompetent $\mathrm{BALB} / \mathrm{c}$ and $\mathrm{C} 57 \mathrm{BL} / 6$ mice suggests that the DFTD cells are immunogenic. To confirm that the failure to establish DFTD xenografts in immunocompetent mice was a specific immune system response, we evaluated antibody, cytokine, and cytotoxic responses in $\mathrm{BALB} / \mathrm{c}$ and $\mathrm{C} 57 \mathrm{BL} / 6$ challenged with viable DFTD cells.

Flow cytometric detection of DFTD cell surface specific antibodies in the serum of $\mathrm{BALB} / \mathrm{c}$ and $\mathrm{C} 57 \mathrm{BL} / 6$ mice provided the 


\section{Cell-mediated cytotoxicity}

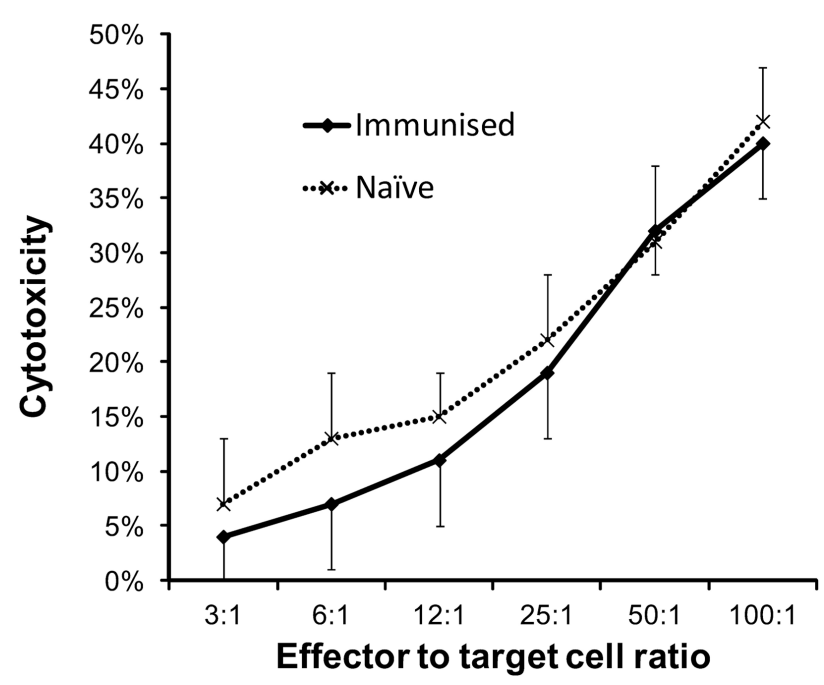

FIGURE 6 | Splenocytes from naïve and immunized C57BL/6 mice were evaluated for cytotoxic responses against DFTD cells. Immunized mice were given IP injections on days 0 and 14 and splenocytes harvested on day 21. DFTD target cells were labeled with CFSE and dead cells identified with PI staining. Flow cytometry was used to calculate cell mediated cytotoxicity as a percentage of CFSE labeled DFTD target cells. Splenocytes from naïve $(n=4)$ or immunized $(n=5)$ mice and DFTD target cells were incubated for $18 \mathrm{~h}$ in vitro at $37^{\circ} \mathrm{C}$ with $5 \% \mathrm{CO}_{2}$. Results represent the mean and standard deviation of the combined cytotoxicities of all mice with four technical replicates for each mouse.

most robust method for detection of an immune response following immunization with DFTD tumor cells. There was no evidence for substantial levels of preformed antibodies as serum from naïve mice showed minimal binding to DFTD cells. This low level of binding could have been due to low concentrations of preformed antibodies, or simply non-specific binding. This suggests that hyperacute xenograft rejection, which depends on preformed antibodies, was unlikely to be the major cause preventing DFTD establishment.

The SC injection route for immunizations had originally been selected because of its similarity to the transfer of DFTD cells in the Tasmanian devil population. By comparing single versus multiple site SC injections, it was established that multiple site injections produced a more intense antibody response. This was most likely due to the targeting of an increased number of draining lymph nodes. Although implantations at a single site may occur frequently in wild Tasmanian devils, multiple site injections would be more effective in a vaccination program. IP injections produced an even greater and more consistent antibody response. The enhanced immunological response via the IP route in the mouse model may have implications for the induction of a protective immune response in Tasmanian devils. IP injections of vaccines for Tasmanian devils may prove more effective than SC injections. Additionally, when DFTD cells need to be inactivated for vaccine trials irradiation is preferable to sonication or freeze/thawing
$B A L B / c$ versus $C 57 B L / 6$ antibody responses

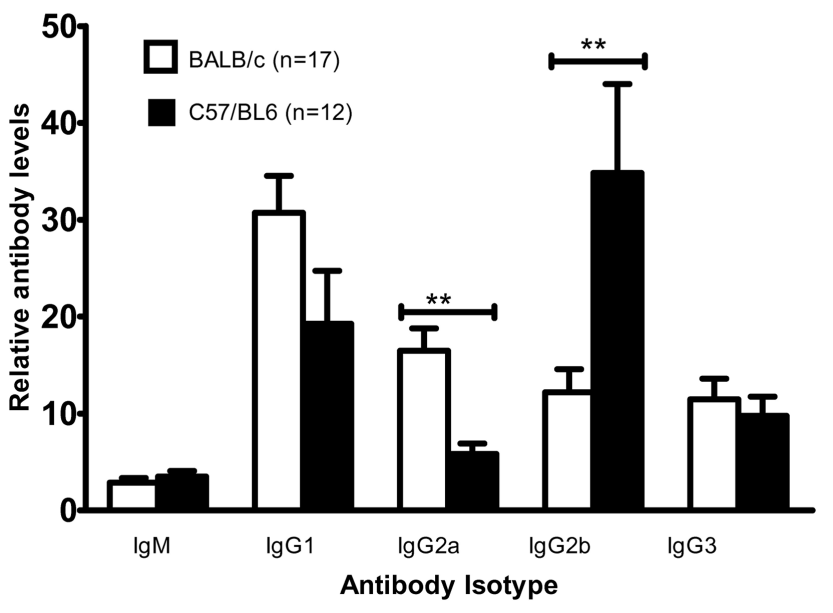

FIGURE 7 | Cohorts of BALB/c and C57BL/6 mice were injected with $10^{6}$ DFTD cells by IP injection on Day 0 , with a second injection between day 14 and 16; serum was collected 7-8 days later. While anti-DFTD antibody expression varied between individuals there was a consistent trend that BALB/c mice were skewed toward higher levels of IgG2a and lower levels of IgG2b compared to C57BL/6 mice (Data are expressed as mean \pm SEM, probability calculated by unpaired Student's t-test, ${ }^{*} P<0.01$ ).

because the irradiated cells retain their immunogenicity in the mouse model and it is reasonable to presume this would translate to the Tasmanian devil's responses.

Intraperitoneal injections generated both $\operatorname{IgM}$ and $\operatorname{IgG}$ responses to the DFTD cells. The switch from IgM to IgG was detected 4 and 7 days after the first immunization with DFTD cells. This is consistent with a T cell dependent antibody response to any cellular antigen. Secondary responses after a second DFTD immunization following a 95-day rest revealed an amplified IgG response. Consequently antibody responses to DFTD cells are characteristic of a typical $\mathrm{T}$ cell dependent humoral immune response to a cellular antigen.

The cell mediated arm of the immune response was evaluated with an in vitro cytotoxicity assay. Splenocytes from DFTD immunized mice produced the same level of cytotoxicity as splenocytes from naïve mice. This suggests that the response did not benefit from priming of cytotoxic T lymphocytes of the specific immune system. Cytotoxicity may be mediated by NK, NKT cells, or unprimed $\mathrm{T}$ cells responding to xenogeneic determinants. The significance of this finding is twofold. Cytotoxic cells can recognize DFTD cells and they can be killed. The relevance of this latter point is that as DFTD cells are susceptible to killing by murine cells they should also be susceptible to killing by cytotoxic cells of the Tasmanian devil.

Devil Facial Tumour Disease cells do not express surface MHCI molecules (8) and therefore should be targets for NK cells. If NK cells from the mouse are the effector cells, this suggests that DFTD cells express the obligatory activating receptors. We have evidence that Tasmanian devils have NK cells (13). If mouse NK-like cells can bind to and destroy the DFTD cell in vivo then NK cells of 

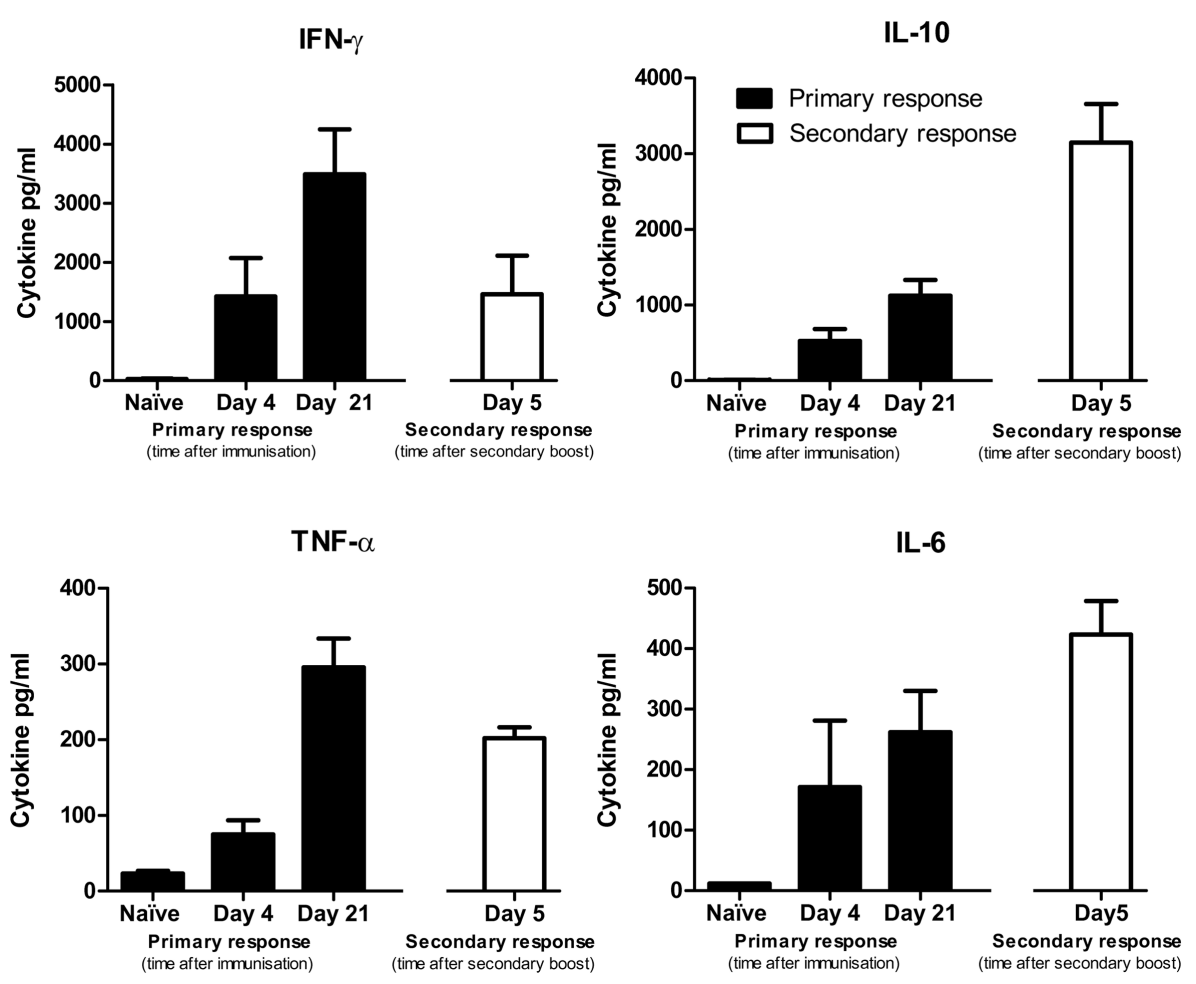

FIGURE 8 | Cytokine production by splenocytes from naïve mice or DFTD immunized mice, cultured in vitro with DFTD cells for $\mathbf{7 2} \mathbf{~ h}$.

Primary responses refer to mice given a single injection of $2 \times 10^{6}$ DFTD cells and day refers to time post immunization. Secondary responses refer to mice immunized with $2 \times 10^{6}$ DFTD cells, rested 57 days, given a second immunization with $2 \times 10^{6}$ DFTD cells and splenocytes collected 5 days later. Data expressed as mean of five mice \pm SEM except naïve responses $(n=3)$. the Tasmanian devil should also have the capacity to kill DFTD. The mechanism by which the DFTD cells inhibit or circumvent the NK cells of Tasmanian devils following allograft transmission of DFTD remains unknown.

With regards to NKT cells, they do not require MHC-I molecules for binding but are activated by glycolipid antigens binding to CD1d $(14,15)$. We have previously stated that $\beta_{2}$-microglobulin is down-regulated, which is an obligatory molecule associated CD1d molecules making NKT cells unlikely effector cells. However, as $\beta_{2}$-microglobulin expression can be restored in the presence of cytokines (8), NKT cells could have contributed to the immune response. It is not currently known if DFTD cells express glycolipid antigens capable of activating NKT cells.

The isotype switching of $\mathrm{B}$ cell antibody production is $\mathrm{T}$ cell dependent and directed by $\mathrm{T}$ cell derived cytokines resulting in antibody isotypes characteristic of either a $\mathrm{T}_{\mathrm{H}} 1$ or $\mathrm{T}_{\mathrm{H}} 2$ profile (16-18). Although an immune response against DFTD cells was demonstrated, we considered the possibility that DFTD could be a tumor cell line that biases the immune response to a $\mathrm{T}_{\mathrm{H}} 2$ response. This would permit tumor surveillance escape by suppressing $\mathrm{T}_{\mathrm{H}} 1$ anti-tumor responses $(9,19)$. This was analyzed by evaluating cytokines. IFN- $\gamma$, characteristic of a $\mathrm{T}_{\mathrm{H}} 1$ response (20), was upregulated within days of immunization with DFTD cells. This was followed by a strong and persistent up-regulation of IL-10, which is a $\mathrm{T}_{\mathrm{H}} 2$ type cytokine that counterbalances the $\mathrm{T}_{\mathrm{H}} 1$ responses generated by IFN- $\gamma(20)$. IL-10 also promotes the activation and proliferation of antigen-specific B cells (9). The timing of IL-10 up-regulation is also consistent with the detection of IgG antibodies against DFTD cells in the mice. As IgG isotypes discriminate between $\mathrm{T}_{\mathrm{H}} 1$ and $\mathrm{T}_{\mathrm{H}} 2$ responses (20), these were evaluated in the $\mathrm{BALB} / \mathrm{c}$ and C57BL/6 mice. Inoculation with DFTD cells did not skew the immune response of either strain toward a $\mathrm{T}_{\mathrm{H}} 1$ or $\mathrm{a}_{\mathrm{H}} 2$ response. There was some skewing toward IgG2a in the BALB/c mice and toward IgG2b in the C57BL/6 mice. Both strains of mice expressed high levels of IgG1, which is regarded as a $\mathrm{T}_{\mathrm{H}} 2$ response. The relevance of these findings is that the DFTD cells do not consistently bias the immune response toward a non-protective $\mathrm{T}_{\mathrm{H}} 2$ response.

As with results derived from any animal or in vitro model, there will be limitations and the immunological mechanisms observed may not translate to the natural host species. The combined cytokine and antibody profile in these mice demonstrates a humoral response to DFTD, which is consistent with xenograft rejection mechanisms involving NK cells (21). Xenograft rejections are MHC-I independent and allograft rejections are based upon MHC-I incompatibilities. DFTD cells do not express surface MHC-I (8) and therefore cannot be rejected by MHC incompatibilities. Rejection must therefore rely on other cell types such as NK cells or NKT cells. NK cells are the most likely cell type involved in the rejection of the DFTD xenograft described in this 

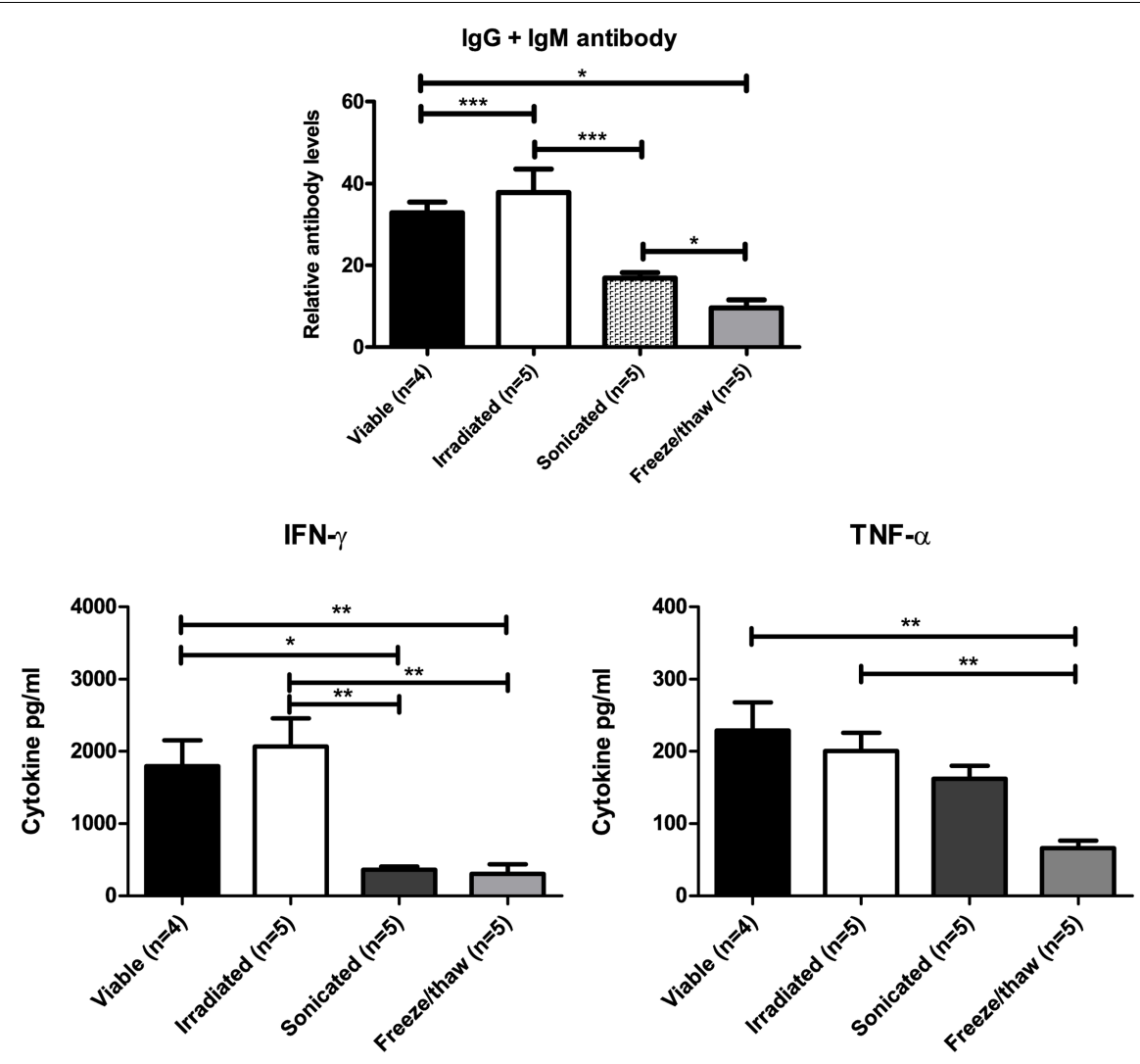

FIGURE 9 | Mice immunized with sonicated or freeze/thaw DFTD cells produced lower levels of anti-DFTD antibody and lower levels of IFN- $\gamma$ and TNF- $\alpha$ compared to mice immunized with viable or

irradiated DFTD cells (Data are expressed as mean \pm SEM, probability calculated by unpaired Student's $t$-test, ${ }^{*} P<0.05,{ }^{*} P<0.01$, and *** $P<0.001$ ).

paper. Clearly, Tasmanian devil NK or NKT cells are not targeting the DFTD tumor cells. But as mouse cells can target DFTD, immunogenic membrane signals would be present to act as targets.

In conclusion, the main finding of this study is that DFTD cells are suitable targets for immunotherapy. There is a need to develop a vaccine or treatment to prevent extinction of the Tasmanian devil in the wild and various strategies can be trialed in the mice before moving to clinical trials with Tasmanian devils.

\section{ACKNOWLEDGMENTS}

Research support was provided by the Australian Research Council, Dr. Eric Guiler Tasmanian devil research grant and the Select Foundation. The funders had no role in study design, data collection and analysis, decision to publish or preparation of the manuscript. The authors wish to thank Narelle Phillips, Mark Cozens, Cesar Tovar and Alexandre Kreiss for technical assistance. We thank Anne-Maree Pearse and Kate Swift of DPIPWE for the provision of DFTD cell lines. We are grateful to the staff from The University of Tasmania Central Animal House, especially Angela Maher for the care and breeding of the mice used in our research.

\section{REFERENCES}

1. Murchison EP, Schulz-Trieglaff OB, Ning Z, Alexandrov LB, Bauer MJ, Fu B, et al. Genome sequencing and analysis of the Tasmanian devil and its transmissible cancer. Cell (2012) 148(4):780-91. doi:10.1016/j.cell.2011.11.065
2. Jones ME, Paetkau D, Geffen E, Moritz C. Genetic diversity and population structure of Tasmanian devils, the largest marsupial carnivore. Mol Ecol (2004) 13(8):2197-209. doi:10.1111/j.1365-294X.2004.02239.x

3. Siddle HV, Kreiss A, Eldridge MDB, Noonan E, Clarke CJ, Pyecroft S, et al. Transmission of a fatal clonal tumor by biting occurs due to depleted MHC diversity in a threatened carnivorous marsupial. Proc Natl Acad Sci U S A (2007) 104(41):16221-6. doi:10.1073/pnas.0704580104

4. Siddle HV, Kaufman J. A tale of two tumours: comparison of the immune escape strategies of contagious cancers. Mol Immunol (2013) 55(2):190-3. doi:10.1016/j.molimm.2012.10.017

5. Murchison EP. Clonally transmissible cancers in dogs and Tasmanian devils. Oncogene (2008) 27(Suppl 2):S19-30. doi:10.1038/onc.2009.350

6. Murchison EP, Tovar C, Hsu A, Bender HS, Kheradpour P, Rebbeck CA, et al. The Tasmanian devil transcriptome reveals Schwann cell origins of a clonally transmissible cancer. Science (2010) 327(5961):84-7. doi:10.1126/science. 1180616

7. Pearse AM, Swift K. Allograft theory: transmission of devil facial-tumour disease. Nature (2006) 439(7076):549. doi:10.1038/439549a

8. Siddle HV, Kreiss A, Tovar C, Yuen CK, Cheng YY, Belov K, et al. Reversible epigenetic down-regulation of MHC molecules by devil facial tumour disease illustrates immune escape by a contagious cancer. Proc Natl Acad Sci U S A (2013) 110(13):5103-8. doi:10.1073/Pnas.1219920110

9. Singh A, Qin H, Fernandez I, Wei J, Lin J, Kwak LW, et al. An injectable synthetic immune-priming center mediates efficient T-cell class switching and T-helper 1 response against B cell lymphoma. J Control Release (2011) 155(2):184-92. doi:10.1016/j.jconrel.2011.06.008

10. Mills CD, Kincaid K, Alt JM, Heilman MJ, Hill AM. M-1/M-2 macrophages and the Th1/Th2 paradigm. J Immunol (2000) 164(12):6166-73. doi:10.4049/ jimmunol.164.12.6166 
11. Reiner SL, Locksley RM. The regulation of immunity to Leishmania major. Annu Rev Immunol (1995) 13:151-77. doi:10.1146/annurev.iy.13.040195.001055

12. Kreiss A, Tovar C, Obendorf DL, Dun K, Woods GM. A murine xenograft model for a transmissible cancer in Tasmanian devils. Vet Pathol (2011) 48(2):475-81. doi: $10.1177 / 0300985810380398$

13. Brown GK, Kreiss A, Lyons AB, Woods GM. Natural killer cell mediated cytotoxic responses in the Tasmanian devil. PLoS One (2011) 6(9):e24475. doi:10.1371/journal.pone.0024475

14. Joyce S, Girardi E, Zajonc DM. NKT cell ligand recognition logic: molecular basis for a synaptic duet and transmission of inflammatory effectors. J Immunol (2011) 187(3):1081-9. doi:10.4049/jimmunol.1001910

15. Godfrey DI, Rossjohn J. New ways to turn on NKT cells. J Exp Med (2011) 208(6):1121-5. doi:10.1084/jem.20110983

16. Isakson PC, Pure E, Vitetta ES, Krammer PH. T-cell-derived B-cell differentiation factor(s) - effect on the isotype switch of murine B-cells. J Exp Med (1982) 155(3):734-48. doi:10.1084/jem.155.3.734

17. Kanai N, Min W-P, Ichim TE, Wang H, Zhong R. Th1/Th2 xenogenic antibody responses are associated with recipient dendritic cells. Microsurgery (2007) 27(4):234-9. doi:10.1002/micr.20342

18. Tangye SG, Ferguson A, Avery DT, Ma CS, Hodgkin PD. Isotype switching by human B cells is division-associated and regulated by cytokines. J Immunol (2002) 169(8):4298-306. doi:10.4049/jimmunol.169.8.4298

19. Salazar-Onfray F. Interleukin-10: a cytokine used by tumors to escape immunosurveillance. Med Oncol (1999) 16(2):86-94. doi:10.1007/bf02785841
20. Schulte S, Sukhova GK, Libby P. Genetically programmed biases in Th1 and Th2 immune responses modulate atherogenesis. Am J Pathol (2008) 172(6):1500-8. doi:10.2353/ajpath.2008.070776

21. Azimzadeh A, Meyer C, Ravanat C, Cazenave JP, Wolf P. Xenograft rejection: molecular mechanisms and therapeutic prospects. Hematol Cell Ther (1996) 38(4):331-43. doi:10.1007/s00282-996-0331-2

Conflict of Interest Statement: The authors declare that the research was conducted in the absence of any commercial or financial relationships that could be construed as a potential conflict of interest.

Received: 17 February 2014; accepted: 13 May 2014; published online: 27 May 2014. Citation: Pinfold TL, Brown GK, Bettiol SS and Woods GM (2014) Mouse model of Devil Facial Tumour Disease establishes that an effective immune response can be generated against the cancer cells. Front. Immunol. 5:251. doi: 10.3389/fimmu.2014.00251 This article was submitted to Tumor Immunity, a section of the journal Frontiers in Immunology.

Copyright (C) 2014 Pinfold, Brown, Bettiol and Woods. This is an open-access article distributed under the terms of the Creative Commons Attribution License (CC BY). The use, distribution or reproduction in other forums is permitted, provided the original author(s) orlicensor are credited and that the original publication in this journal is cited, in accordance with accepted academic practice. No use, distribution or reproduction is permitted which does not comply with these terms. 